\title{
De-Noising Corrupted ECG Signals By Empirical Mode Decomposition (EMD) With Application of Higher Order Statistics (HOS)
}

\author{
Mitra DJ ${ }^{1}$, Shahjalal $\mathrm{M}^{2}$, Kiber MA ${ }^{3}$ \\ ${ }^{1.3}$ Department of Applied Physics, Electronics and Communication Engineering, University of Dhaka, \\ Bangladesh \\ ${ }^{2}$ Departments of Basic Science, Primeasia University, Banani, Dhaka, Bangladesh
}

\begin{abstract}
The electrocardiogram (ECG) signals which are extensively used for heart disease diagnosis and patient monitoring are usually corrupted with various sources of noise. In this paper, an algorithm is developed to de-noise ECG signals based on Empirical Mode Decomposition (EMD) with application of Higher Order Statistics (HOS). The algorithm is applied on several ECG signals for different levels of Signal to Noise Ratio $(S N R)$. The SNR improvement $\left(S N R_{i m p}\right)$ and Percent Root mean square Difference $(P R D(\%))$ are analyzed. The results show that the developed algorithm is a reasonable one to de-noise ECG signals.
\end{abstract}

Keywords: ECG, Empirical Mode Decomposition (EMD), Higher Order Statistics (HOS), Intrinsic Mode Function (IMF)

\section{Introduction}

Biomedical signals reflect the nature and activities of physiological processes. The electrocardiogram (ECG) is the electrical manifestation of the contractile activity of the heart. The ECG is essential for diagnosis, and therefore management of abnormal cardiovascular activity. Noise or unwanted signal is always present in ECG signal, which makes it difficult to analyze. The typical sources of noise are, high frequency noise, motion artifacts in ECG, maternal interference in fetal ECG, EMG noise, instrumentation noise etc. Therefore denoising the ECG signal is a pre-requisite to arrive at proper diagnosis by analyzing it.

A number of methods have been applied to de-noise ECG signals such as, digital filters, ICA, PCA, adaptive filtering, wavelet transform etc. The existing de-noising techniques have certain limitations. The filter bank based de-noising process smoothes the P and R amplitude of the ECG signal, and it is more sensitive to different levels of noise [1]; The statistical model derived in PCA, ICA is not only fairly arbitrary but also extremely sensitive to small changes in either the signal or the noise unless the basis functions are trained on a global set of ECG beat types, moreover, the ICA doesn't allow the prior information about the signals for efficient filtering; adaptive filtering requires reference signal information for the effective filtering process, and the reference signal has to be additionally recorded together with ECG [2]. Wavelets need a basis function to be specified, moreover, the hard-thresholding WT leads to oscillation of the reconstructed ECG signal, and the soft-thresholding method reduce the amplitudes of the ECG waveform, especially reduce the amplitudes of the $\mathrm{R}$-waves which is more important to diagnose the heart diseases [3].

In this paper, an algorithm has been developed to de-noise ECG signal based on Empirical Mode Decomposition (EMD) along with application of Higher Order Statistics (HOS). The EMD method is totally adaptive and data driven. This method doesn't need a-priori basis function selection (i.e. mother wavelet) for signal decomposition. EMD is effectively used for signal de-noising in a wide range of applications, such as acoustic signals, ionospheric signals, in the study of heart rate variability (HRV), analysis of respiratory mechanomyographic signals, crackle sound analysis in lung sounds and enhancement of cardiograph signals. The acceptance of the method as a processing tool is stressed by the large number of publications in diverse areas of signal processing including financial applications, fluid dynamics, ocean engineering and electromagnetic field time series analysis [4]. Thus EMD is a versatile method to de-noise and analyze nonstationary signals.

\section{Empirical Mode Decomposition (Emd)}

Empirical Mode Decomposition (EMD) method is a new non-linear technique, which was first formulated by Dr. Norden Huang of NASA in 1996, for adaptively representing non-stationary signals as sums of zero mean AM-FM components [5]. This method has very good results in the analysis of non-linear and nonstationary signals, especially to the exact representation of the energy of the signal and the frequency content thereof in relation to time. The main feature of the new method is to analyze the signal in structural components known as Intrinsic Mode Functions (IMFs), arising from the signal itself and not defined in advance, so that the 
analysis can be considered not as a-priori, but instead a-posteriori. The final result is the ability to display the spectrum of the signal in function of time, much more accurately than the traditional methods. An Intrinsic Mode Function (IMF) represents the oscillating mode embedded in the original data. IMF is a function that satisfies the following two conditions:

1. The total number of local extrema and the number of zero crossings should be equal to each other or differ by at most 1 .

2. At any point the mean of upper and lower envelopes respectively defined by local maxima and the local minima should be zero.

\subsection{Sifting Process: Extraction of IMF by EMD}

The algorithm is as following [5]:

Step 1: For a signal $x(t)$, create upper envelope $E_{\max }(t)$ by local maxima and lower envelope $E_{\text {min }}(t)$ by local minima by using cubic splines interpolation.

Step 2: Calculate the mean of the upper and lower envelope:

$m_{1}=\frac{E_{\max }(t)+E_{\min }(t)}{2}$

Step 3: Subtract the mean from original data:

$h_{1}(t)=x(t)-m_{1}(t)$

Step 4: Verify that $h_{1}(t)$ satisfies conditions for IMFs. If not, repeat steps 1-4 and the new balance emerges as:

$h_{11}(t)=h_{1}(t)-m_{11}(t)$

Where, $m_{11}(t)$ is the mean value of the envelopes defined by the extremes of $h_{1}(t)$.

Step 5: Get first IMF (after k iterations):

$h_{1 k}(t)=h_{1(k-1)}(t)-m_{1 k}(t)$

Where, $k$ is the number of repetitions until the first IMF, $h_{1 k}(t)=I M F_{1}$, occurs.

Step 6: Calculate first residue:

$r_{1}(t)=x(t)-h_{1 k}(t)$

Step 7: Repeat whole algorithm with $r_{l}(t), r_{2}(t) \ldots$ until residue is monotonic function.

Step 8: After $n$ iterations $x(t)$ is decomposed according to equation,

$x(t)=\sum_{i=1}^{n} I M F_{i}+r_{n}$

\section{Higher Order Statistics (HOS)}

The use of higher-order statistics provides insight into signals which is not always available at lower orders. Additionally, Gaussian-distributed signals have the interesting characteristic of disappearing at higher orders. Because so much of the noise and interference environment is Gaussian-distributed, higher order statistics thus offer the promise of a useful method of noise reduction [6]. ECG signal is easily contaminated by different sources of noises, as mentioned previously. Different noise sources can be approximated by a white Gaussian noise source [7]. So, after EMD is used to decompose the ECG signal into its IMF, higher order statistics is a good choice to remove any Gaussian scales from the signal. In this paper, Kurtosis and Bispectrum of every IMF are used as HOS parameters to check the Gaussianity, which is then followed by Bootstrap technique.

In probability theory and statistics Kurtosis is any measure of the peakedness of the probability distribution of a real-valued random variable. Kurtosis is defined as the normalized version of the fourth order Cumulant. Assuming a zero mean signal, the normalized Kurtosis is expressed as:

$K_{4}=\frac{E\left\{x(t)^{4}\right\}}{\left\{E\left[x(t)^{2}\right]\right\}^{2}}-3=N \frac{\sum_{n=1}^{N} x(n)^{4}}{\left(\sum_{n=1}^{N} x(n)^{2}\right)^{2}}-3$

Where $\mathrm{N}$ is the number of signal samples, and $\mathrm{n}=1,2, \ldots . \mathrm{N}$

And, a Kurtosis estimator can take values as:

$\left|K_{4}\right| \leq \frac{\sqrt{\frac{24}{N}}}{\sqrt{1-a}}$

Where $a$ is as authorized confidence percentage value, with a numerically estimated optimum equal to $90 \%$. However, even though the Kurtosis of a Gaussian signal is restricted by equation (8), in fact Kurtosis estimation may still be invalid, especially when the samples of the signal are not numerous enough to ensure convergence. In these cases, the solution comes in the form of bootstrap technique.

Bootstrap is a statistical method to increase the accuracy of the estimator, and it is very effective in cases when the available signal samples are limited. Bootstrap does exactly what a scientist do in practice if it's possible: it repeats the experiment many times. Bootstrap randomly reassigns the observations, re-computes the estimates many times and treats these reassignments as repeated experiments [8].

In this study Bootstrap is used to evaluate the Kurtosis of each of the signal IMFs right after EMD. The number of the IMF samples reassignments is limited to 1000 for computational load purposes. The Bootstrap 
algorithm gives a maximum and minimum Kurtosis estimation of the Kurtosis value for each of the signal IMFs, which in turn is compared with the theoretical Kurtosis limit as it is calculated by equation (8).

Signal Bispectrum is another candidate to test Gaussianity. The Bispectrum is defined as the third order Spectrum of the signal and is calculated either as the Fourier transform of its third order Cumulant, or as the triple product of its Fourier coefficients, [9]. That is:

$B_{2}^{x}\left(\omega_{1}, \omega_{2}\right)=E\left\{X\left(\omega_{1}\right) X\left(\omega_{2}\right) X^{*}\left(\omega_{1}+\omega_{2}\right)\right\}=m_{3} X\left(\omega_{1}\right) X\left(\omega_{2}\right) X^{*}\left(\omega_{1}+\omega_{2}\right)$

Where, $\left|\omega_{1}\right| \leq \pi,\left|\omega_{1}\right| \leq \pi,\left|\omega_{1}+\omega_{2}\right| \leq \pi$,

$\mathrm{X}(\omega)$ is the Fourier transform of the signal $\mathrm{x}(\mathrm{n})$, and, $\mathrm{m}_{3}$ is the third order moment.

The Bispectrum of a Gaussian process is zero. However, there exist statistical processes where the Bispectrum is zero despite deviating from Gaussianity. In other words a non Gaussian signal may admit zero Bispectrum, but if a signal is Gaussian its Bispectrum has to be equal to zero. To this end, using the Bispectrum criterion to ensure Gaussianity after the signal is classified as Gaussian by the Kurtosis test, appears to be a good choice. Although the computational cost increases by using two Gaussianity estimators, by doing this it can be ensured that the IMF under examination can be safely classified as Gaussian and excluded from the signal reconstruction process. After the Gaussianity is checked thresholding rule is applied on the non-Gaussian IMFs. The threshold is a modified version of the universal threshold proposed by Dohono [1], expressed as:

$T=c \sqrt{V_{i} 2 \ln (N)}$

Where, $V_{i}$ is the noise variance estimated by the noise model for the $i-t h I M F,(i \geq 2), N$ is the number of signal samples, and $\mathrm{c}$ is a constant experimentally found to take values 1 to 0.7 depending of the type of signal.

The assumption also that the total noise energy is captured by the first IMF is not valid in the general case, therefore the noise variance for the first IMF is estimated using a better estimator as:

$V_{1}=\left(\frac{\text { median }\left|I M F_{1}\right|}{0.6745}\right)^{2}$

An alternative approach for the noise variance estimator is where the absolute median deviation of the first IMF is taken into account as:

$V_{1}=\left(\frac{\text { median }\left(\mid I M F_{1}-\text { median }\left(I M F_{1}\right) \mid\right)}{0.6745}\right)^{2}$

A series of simulations concluded that the second of these two versions of noise variance estimator, performs better for all types of signals.

Then the variance of each of the IMFs can be parameterized as a function of the first IMF variance as:

$V_{i}=\frac{V_{1}}{\beta_{H}} \rho_{H}^{-2(1-H) k}, k \geq 2$

Where, $\beta_{\mathrm{H}}$ is experimentally estimated in for three values of the Hurst exponent as $\left(\mathrm{H}=0.2, \beta_{\mathrm{H}}=0.487\right)$, $\mathrm{H}=0.5, \beta_{\mathrm{H}}=0.719,\left(\mathrm{H}=0.8, \beta_{\mathrm{H}}=1.025\right)$, and, $k \geq 2$ and $\rho_{H} \approx 2$.

Having now determined the thresholds for each IMF, the de-noising method would necessitate zeroing the portion of the IMF which is below the threshold. However, the IMF nature requires setting to zero the IMF portion between two adjacent zero crossings, when the absolute maximum of the IMF in this interval is below the predefined threshold. This fact is based on the assumption that if the extrema which lies inside two adjacent zero crossings interval exceeds the threshold, the interval is signal dominant; otherwise it is noise dominant [10].

The thresholding operation for the EMD case and for every two successive zero crossings interval, $z_{i}^{j}=$ $\left[z_{i}^{j}, z_{i}^{j+1}\right]$, the hard-thresholding can be expressed as:

$\tilde{z}_{i}^{j}=\left\{\begin{array}{cc}z_{i}^{j}, & \left|r_{i}^{j}\right|>T_{i} \\ 0, & \left|r_{i}^{j}\right| \leq T_{i}\end{array}\right.$

Where, $\tilde{z}_{i}^{j}$ denotes the thresholding interval, $i$ is the IMF order index, $r_{i}^{j}$ is the $j^{\text {th }}$ extrema of the $i^{\text {th }}$ IMF, and $\mathrm{j}$ $=1,2, \ldots,\left(M_{i}-1\right)$, with $M_{i}$ being the number of the zero-crossings of the $i^{\text {th }}$ IMF.

For the soft-thresholding cases $\tilde{z}_{i}^{j}$ is given as:

$\tilde{z}_{i}^{j}= \begin{cases}z_{i}^{j} \frac{\left|r_{i}^{j}\right|-T_{i}}{r_{i}^{j}}, & \left|r_{i}^{j}\right|>T_{i} \\ 0, & \left|r_{i}^{j}\right| \leq T_{i}\end{cases}$

The thresholded IMF is formed by concatenating the thresholded intervals as:

$\widetilde{I M F_{i}}=\left[\tilde{z}_{i}^{1}, \tilde{z}_{i}^{2}, \tilde{z}_{i}^{3}, \quad \tilde{z}_{i}^{j}\right]$ 


\section{Proposed Algorithm to De-Noise ECG Signal}

The main objects to suppress noise from an ECG signal are,

(a) Improving the signal to noise ratio (SNR), in order to unambiguously distinguish the characteristics of the signal.

(b) Non-alteration of the original waveform shape and especially that of the complex QRS, preventing deformation waves $\mathrm{P}$ and $\mathrm{T}$, as well as maintaining proper ST area, so as maintain visibility of signal T.

Regarding these facts, the steps to de-noise ECG signal are as following,

Step 1: Empirical Mode Decomposition (EMD) of the ECG signal

Step 2: Delineation and separation of QRS complex

Step 3: Preservation of the QRS complex by using proper window

Step 4: Suppression of noise from intermediate portions of QRS complexes

Step 5: Checking Gaussianity of IMFs by higher order statistics (HOS)

Step 6: Thresholding the non-Gaussian IMFs and reconstruction of the ECG signal

\section{Implementation of The Algorithm}

The developed algorithm is applied on ECG signals, which were taken from the Department of Biomedical Physics and Technology, University of Dhaka. To observe the versatility of the technique the algorithm is implemented on ECG signals of all 12 leads, and for different levels of input SNR. Fig. 1 and 2 show an uncorrupted signal and corrupted noisy signal. The noisy signal is decomposed into IMFs, which is shown in Fig. 3.

The basic principle of de-noising by EMD is to represent the de-noised signal with a partial sum of the IMFs. Although various approaches have been proposed to identify whether a specific IMF contains useful information or noise, their performances are not satisfactory when directly applied to the problem of ECG denoising, as discussed next.

Examining the IMFs in Fig 3, it's easy to find that the IMF1 contains almost nothing but high frequency noise, and that the rest IMFs can be considered to mainly contain useful information about the ECG components, except the IMF2 which contains both high frequency noise and components of the QRS complex. Here comes the dilemma. If the IMF1 is simply discarded as noise, the output will still consist of considerable noise as illustrated in Fig. 4(a). If the IMF2 is removed together, the resultant ECG will have the R waves heavily distorted as shown in Fig. 4(b). Therefore, neither result is satisfactory.

The rate of information change in the QRS complex is very high compared to that of the other parts of an ECG signal. An analysis of the EMD on clean and noisy ECG indicates that the QRS information is mainly embedded in the first three high frequency IMFs. As a consequence, in a noisy case, a desirable approach to de-noise the corrupted ECG signal $y[n]$ in the EMD domain would be to filter out the noisy parts of the first three IMFs without discarding the IMFs completely thus preserving the QRS complex. Now, adding first three IMFs: $d[n]=$ IMF1 + IMF2+ IMF3 is obtained from the corresponding ECG signal. Fig. 5 presents the original uncorrupted and noisy ECG signals and the respective plots of $d[n]$ in each case. It is revealed from this figure that the oscillatory pattern of the QRS complex, and that of the $d[n]$ in the QRS complex region are highly similar to each other. So, QRS complex portion is the least affected part of ECG by noise.

So, the algorithm to delineate QRS complex is,

Step 1: Identify the fiducial points, which is the peaks of the R-wave

Step 2: Sum the first three IMFs to obtain $d[n]$

Step 3: Find the two nearest local minima on both sides of the fiducial point

Step 4: Detect the two closest zero-crossing points on the left-hand side of the left minimum and on the righthand side of the right minimum. These two points are identified as boundaries of the QRS complex. Next, a window function is designed to preserve the QRS complex. The window function is a time domain window applied to the sum of the first three IMFs, $d[n]$. A general design guideline for the QRS preserving window function is that it should be flat over the duration of the QRS complex and decay gradually to zero so that a smooth transition introduces minimal distortion. In this work, Tukey window is used. Fig. 6 shows the signal $d[n]$ after windowing operation.

Then the noise in the intermediate portions is suppressed by Savitzky-Golay(S-G) filter. S-G filter is used because of its good reported processing of White Gaussian noise. Now, the Gaussianity of the IMFs is checked according to HOS parameters discussed in section 4. The result of application of HOS is shown in Fig. 7. IMF 4 and 5 pass both the test of Gaussianity and therefore discarded. The non-Gaussian IMFs of index higher than 3 are then Thresholded according to the rules discussed in section 3.

The thresholded non-Gaussian IMFs of index higher than 3 are added with the filtered signal to reconstruct the ECG signals. 


\section{Result and Discussion}

The performance measures are as following:

(a) Improvement in Signal to Noise Ratio, $S N R_{i m p}=10 \log _{10} \frac{\sum_{n=1}^{N}|y[n]-x[n]|^{2}}{\sum_{n=1}^{N}|\hat{x}[n]-x[n]|^{2}}$

Where, $x[n]$ denotes the original ECG signal,

$y[n]$ denotes the noisy ECG signal, and

$\hat{x}[n]$ denotes the reconstructed de-noised ECG signal

(b) Percent Root Mean Square Difference, $P R D=\sqrt{\frac{\sum_{n=1}^{N}(\hat{x}[n]-x[n])^{2}}{\sum_{n=1}^{N} x^{2}[n]}} \times 100$

The mean values of the measures for different values of input SNR, for both soft and hard-thresholding definition for all 12 leads are shown in the table.

The results show that the output SNR of the ECG signals is reasonably improved by de-noising by implementing the developed algorithm. The PRD is also decreased to a satisfactory value, which reveals the applicability of the algorithm in real-world environment.

\section{Conclusion}

EMD is a very effective method to decompose non-stationary signals. However, when it comes to denoise ECG signal, direct de-noising can't be done because it degrades the quality of the signal. In this paper, an algorithm is developed to de-noise ECG signal using EMD along with HOS. The algorithm has been proved to be quite good-performing. It is required to implement the algorithms for signals in noisy environment of the real world. If the developed algorithm can de-noise the noisy signals successfully enough, then the algorithm can be used in micro-controller chips to use the technique in ECG recorder machines through integrated circuits (ICs).

\section{Figures and Table}
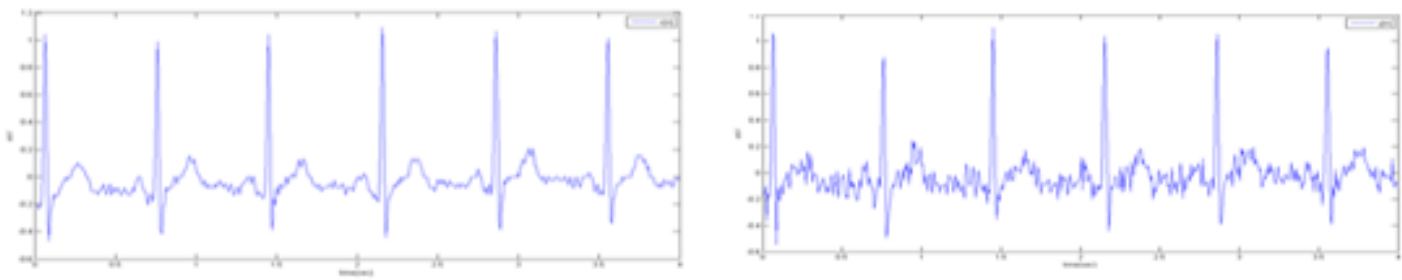

Fig 1: original uncorrupted signal

Fig 2: signal corrupted by noise with SNR $10 \mathrm{db}$
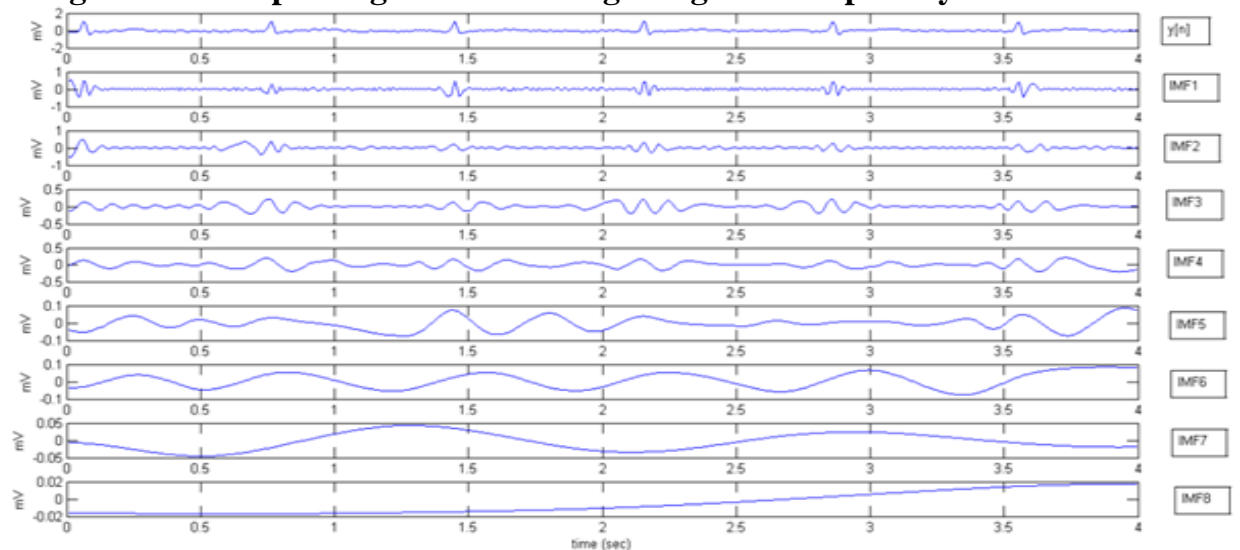

Fig 3: Noisy Signal Decomposed Into Imfs

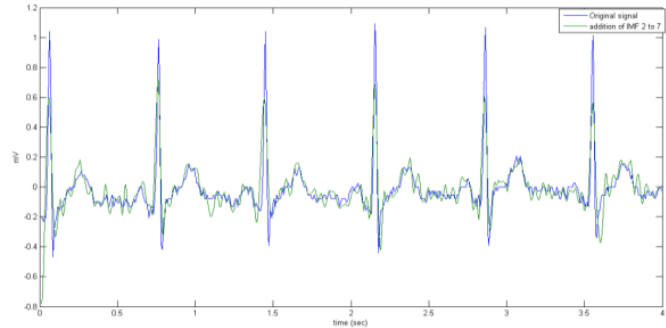

(a)

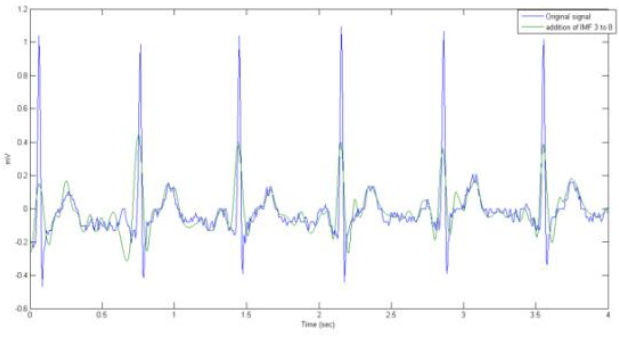

(b)

Fig. 4: direct ECG de-noising (a) removing IMF1 (b) removing IMF1 and IMF2 


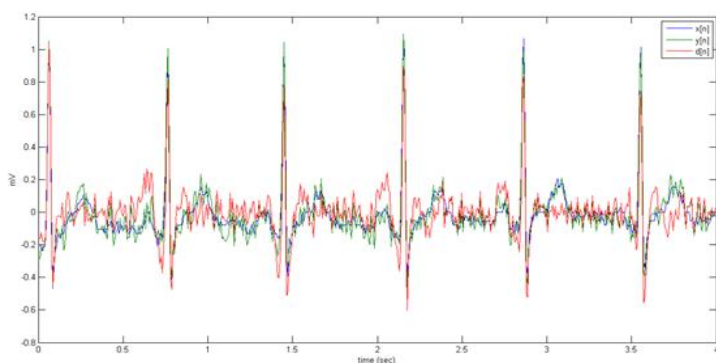

Fig 5: uncorrupted, noisy ECG and $d(n)$

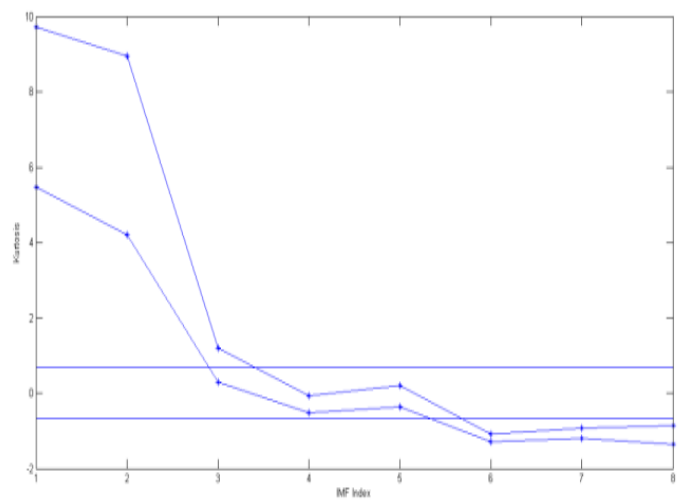

(a)

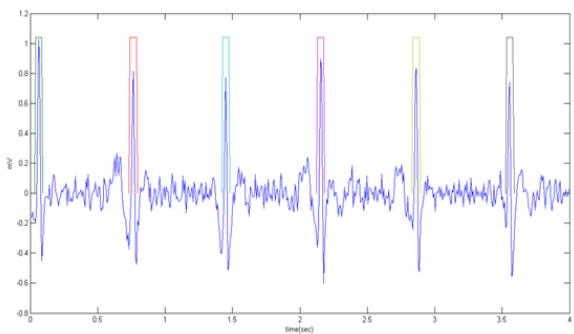

Fig 6: QRS complex preserved by Windowing on $d[n]$

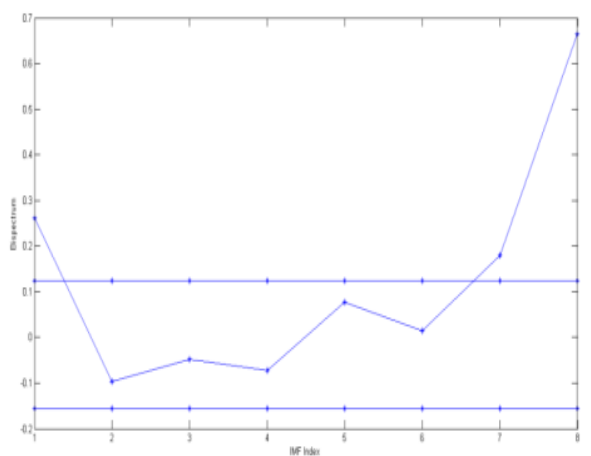

(b)

Fig 7: application of HOS (a) Kurtosis (b) Bispectrum

Table: Results

\begin{tabular}{|l|l|l|l|l|}
\hline $\begin{array}{l}\text { Input } \\
\text { SNR }\end{array}$ & $\begin{array}{l}\boldsymbol{S N R}_{\text {imp }} \text { for } \\
\text { Soft }\end{array}$ & $\begin{array}{l}\boldsymbol{S N R}_{\text {imp }} \\
\text { for Hard }\end{array}$ & $\begin{array}{l}\text { PRD for } \\
\text { Soft }\end{array}$ & $\begin{array}{l}\text { PRD for } \\
\text { Hard }\end{array}$ \\
\hline $\mathbf{5 ~ d b}$ & 3.3308 & 2.9626 & 3.9073 & 4.3065 \\
\hline $\mathbf{1 0 ~ d b}$ & 5.2309 & 3.877 & 2.6595 & 3.2624 \\
\hline $\mathbf{1 5} \mathbf{d b}$ & 10.7506 & 7.7349 & 2.8792 & 3.2624 \\
\hline $\mathbf{2 0} \mathbf{d b}$ & 15.5209 & 12.6355 & 2.7441 & 3.0307 \\
\hline
\end{tabular}

\section{REFERENCES}

[1] L. Sharma, S. Dandapat, and A. Mahanta, Multiscale Wavelet Energies and Relative Energy Based De-noising of ECG Signal, in Communication Control and Computing Technologies, IEEE International Conference , 2010, 491 -495.

[2] T. He, G. Clifford and L. Tarassenko, Application of ICA in Removing Artifacts from the ECG, Neural Processing Letters, 2006, $105-116$.

[3] G. U. Reddy, M. Muralidhar, and S. Varadarajan, EMD De-noising Using Improved Thresholding Based on Wavelet Transform, IJCSNS, 9(9), 2009.

[4] A. Karagiannis, and $\mathrm{Ph}$. Constantinou, On the Processing of White Gaussian Noise Biomedical Signals with the Empirical Mode Decomposition, Biosignal, 2010.

[5] Huang, N. and N. O. Attoh-Okine, The Hilbert-Huang Transform in Engineering (CRC Press, Taylor \& Francis Group6000 Broken Sound Parkway NW, Boca Raton, FL 33487-2742, ISBN-10: 0-8493-3422-5).

[6] D. R. Green, The Utility of Higher Order Statistics in Gaussian Noise Suppression, Naval Postgraduate School, 2003.

[7] P. Flandrin, G. Rilling, and P. Gonsalves, Empirical Mode Decomposition as a Filter Bank.

[8] A.M. Zouhir, and B. Boashash, The Bootstrap and its Application in Signal Processing, IEEE Signal Processing Magazine, 15(1), 1998, 56-76.

[9] A. Al-Smadi, Tests for Gaussianity of a Stationary Time Series, World Academy of Science, Engineering and Technology, 2005.

[10] Y. Kopsinis, and S. McLaughlin, Development of EMD Based De-noising Methods Inspired by Wavelet Thresholding, IEEE Transactions on Signal Processing, 57, 2009, 1351-1362. 